\title{
Comparison of comfort and complications in breast cancer patients of implantable venous access port (IVAP) with ultrasound guided internal jugular vein (IJV) and axillary vein/subclavian vein (AxV/SCV) puncture: a randomized controlled study protocol
}

\author{
Yan-Bo Chen ${ }^{1,2 \#} \wedge$, Hao-Shi Bao ${ }^{1,3 \#}$, He-Ran Deng ${ }^{1,3 \#}$, Ting-Ting Hu ${ }^{1,3}$, Biao-Lin Wen ${ }^{1,3}$, Chun-Yan $\mathbf{Y i}^{1,2}$, \\ Xiu-Wan Chen ${ }^{1,3}$, Li Yan ${ }^{1,4}$, Jian-Nan Wu ${ }^{1,3}$ \\ ${ }^{1}$ Guangdong Provincial Key Laboratory of Malignant Tumor, Epigenetics and Gene Regulation, Sun Yat-sen Memorial Hospital, Sun Yat-sen \\ University, Guangzhou, China; ${ }^{2}$ Department of Orthopedics, Sun Yat-sen Memorial Hospital, Sun Yat-sen University, Guangzhou, China; ${ }^{3}$ Breast \\ Tumor Center, Sun Yat-sen Memorial Hospital, Sun Yat-sen University, Guangzhou, China; ${ }^{4}$ Department of Endocrinology, Sun Yat-sen Memorial \\ Hospital, Sun Yat-sen University, Guangzhou, China \\ Contributions: (I) Conception and design: All authors; (II) Administrative support: None; (III) Provision of study materials or patients: All authors; \\ (IV) Collection and assembly of data: All authors; (V) Data analysis and interpretation: All authors; (VI) Manuscript writing: All authors; (VII) Final \\ approval of manuscript: All authors. \\ \#These authors contributed equally to this work. \\ Correspondence to: Jian-Nan Wu; Li Yan. Yingfeng Road, 33th, Haizhu District, Guangzhou 510000, China. \\ Email: king8702@163.com; hfxyl@163.net.
}

Background Internal jugular vein (IJV) and axillary vein/subclavian vein (AxV/SCV) are commonly used for implantable venous access port (IVAP) implantation in breast cancer (BC) patients with chemotherapy. Previous studies focused on complications between these different approaches and ignored patient comfort. In this study, we aim to compare patient comfort between IJV and AxV/SCV approaches, as well as surgery duration and complications.

Methods: This is a single-center prospective randomized controlled clinical trial. A total of 200 patients diagnosed with invasive BC will be enrolled in this study. After signing written informed consent, patients schedule to undergo IVAP implantation will be randomized at a 1:1 ratio to receive central venous catheters (CVC) with either IJV or AxV/SCV approaches. Baseline as well as demographic data and procedure time of port implantation will be recorded. All patients will receive assessment of comfort with a comfort scale table at days 1, 2 and 7 after implantation surgery. Patients will be followed up and complications will be recorded until devices are removed at the end of the treatment period, or in case of complications. Patient comfort, procedure time of implantation and complications will be compared and analyzed between these two arms.

Discussion: To the best of our knowledge, this is the first study to compare patient comfort as primary outcome measure between IJV and AxV/SCV puncture. This study will further confirm the benefits of ultrasound guidance and may provide a better choice of IVAP implantation for BC patients.

Trial registration: This study has been registered at Chinese Clinical Trial Registry (ChiCTR, www. chictr.org.cn) and Chinese Ethics Committee of Registering Clinical Trials (No. ChiCTR2000034986).

Keywords: Breast cancer (BC); implantable venous access port (IVAP); internal jugular vein (IJV); axillary vein/ subclavian vein (AxV/SCV); comfort; complications

^ ORCID: 0000-0002-8451-4046. 
Submitted Aug 31, 2020. Accepted for publication Oct 26, 2020.

doi: 10.21037/apm-20-1752

View this article at: http://dx.doi.org/10.21037/apm-20-1752

\section{Introduction}

Breast cancer (BC) is the most common malignancy and causes the most cancer deaths in females (1). Systemic chemotherapy is recommended to most of invasive BC patients to reduce the risk of recurrence, with the exception of a few who are at low risk of recurrence $(2,3)$. Administration of chemotherapy through peripheral veins is a dangerous and morbid procedure because of severe side effects in cases of extravasation of chemotherapy drugs, as well as unacceptable pain and psychological trauma $(4,5)$. Consequently, infusion ports with long-term central venous catheters (CVC) are commonly used in BC patients undergoing chemotherapy since their convenience and safety (6).

The most common puncture sites of CVC are internal jugular veins (IJVs) and subclavian veins (SCVs) (7). As a matter of fact, axillary vein $(\mathrm{AxV})$, a direct continuation of the $\mathrm{SCV}$, is also considered as an alternative choice of CVC insertion (8-10). $\mathrm{AxV}$ and SCV are bounded by the lateral margin of the first rib, but to date, experience with $\mathrm{AxV}$ is limited to small case series compared with SCV or IJV. Ultrasound guidance is now considered the gold standard technique for CVC insertion via the IJV or SCV $(11,12)$. Compared with guiding by anatomical landmark, ultrasound guidance has been proved to reduce complications and improve patient comfort (13).

Previous studies have focused on comparing the safety and complications between puncture through IJV and SCV or $\mathrm{AxV}(10,14,15)$. However, patients' comfort has usually been ignored. In this work, we aim to compare the comfort and safety of BC patients punctured through $\mathrm{IJV}$ or $\mathrm{AxV} /$ $\mathrm{SCV}$ with ultrasound guidance, as well as surgery duration and complications. We present the following article in accordance with the SPIRIT reporting checklist (available at http://dx.doi.org/10.21037/apm-20-1752) (16,17).

\section{Methods}

\section{Patient selection}

Participants meet all the following inclusion criteria will be included in our study: (I) age between 18 and 70 years old; (II) have a confirmed histological diagnosis of invasive
BC; (III) have indications for chemotherapy according to the NCCN guideline; (IV) written informed consent. Patients with one or more of these following situations will be excluded: (I) confirmed distant metastasis; (II) diagnosed with anxiety or depression with Self-Rating Anxiety Scale (SAS) and Self-Rating Depression Scale (SDS) questionnaire $(18,19)$; (III) have a history of deep venous thrombosis (DVT) or other coagulation disorders; (IV) are not able to understand the comfort scale questionnaire or cooperate with our work.

\section{Study design}

This is a single-center prospective randomized controlled study. After signing written informed consent, patients will be randomly and equally allocated at a 1:1 ratio in IJV group or AxV/SCV group. A randomization chart will be generated using Microsoft Excel. Randomization will be performed by one of the researchers who will not make operations for patients. We will prepare opaque, sealed envelopes each containing a slip of paper with a computergenerated description of whether the patients will be assigned to IJV group or AxV/SCV group. To exclude the influence of different operators on the study results, all included patients will be implanted with the infusion ports by one of the two designated highly trained doctors (Dr. HSB or Dr. HRD, who are both doctors of Breast Tumor Center in our hospital and major participants in this study). BC patients who are about to receive chemotherapy in our hospital, Sun Yat-sen Memorial Hospital, Sun Yatsen University will be screened and enrolled in our study. All enrolled patients will complete preoperative tests, including complete blood cell count, coagulation screening, electrocardiograph, and puncture vascular ultrasound before port implantation and port removal. All patients will be followed up until devices are removed at the end of the treatment period, or in case of complications such as DVT, port-related infection, tip dislocation, loss of potency or catheter rupture. Study process is showed in Figure 1.

\section{Sample size}

The primary objective of this study is to compare patient 


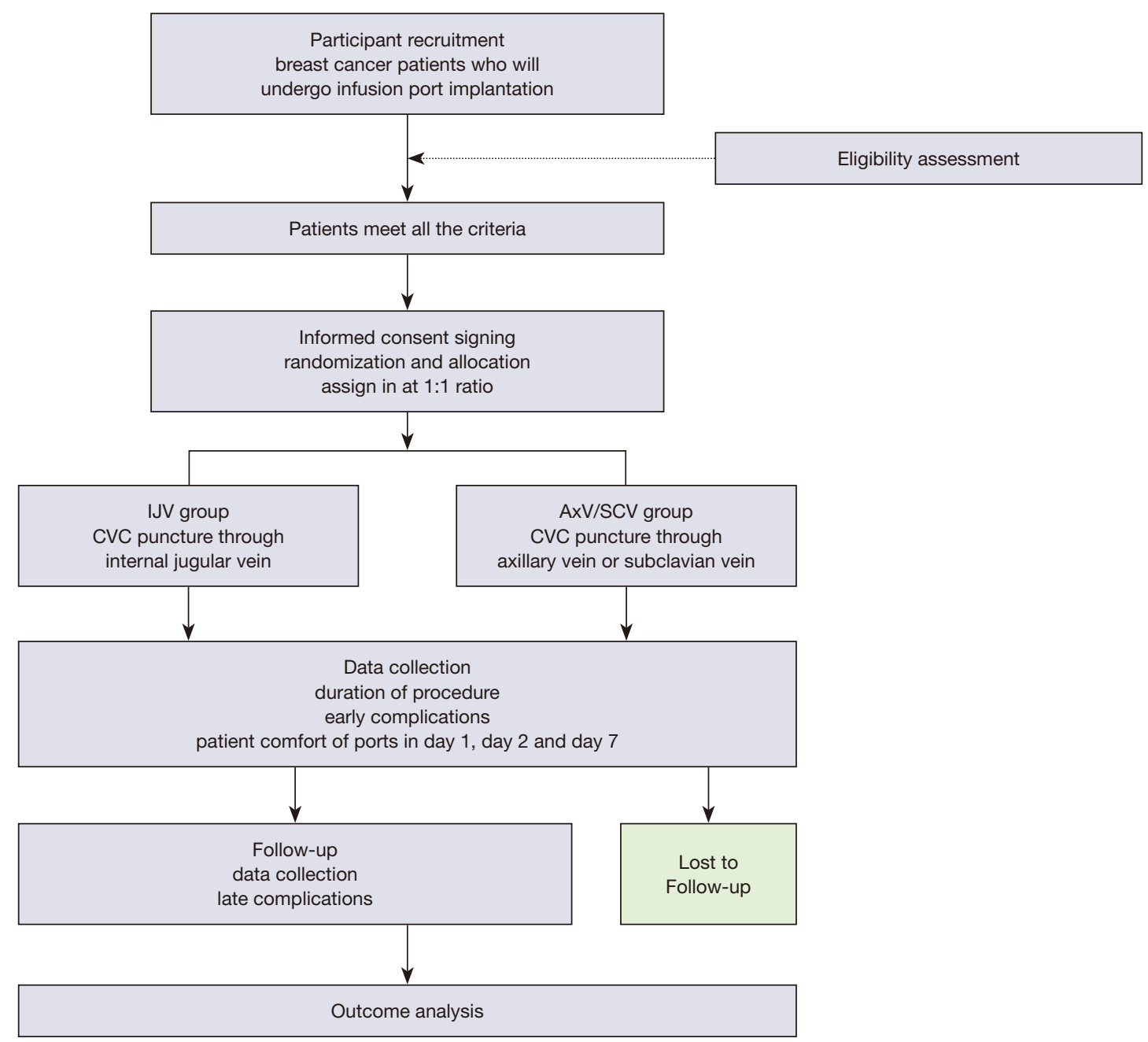

Figure 1 Flow diagram of the progress through the study. IJV, internal jugular vein; CVC, central venous catheters; AxV, axillary vein; SCV, subclavian vein.

comfort between IJV group and AxV/SCV group. A preliminary study with a small amount of cases (30 cases) indicated that patient comfort scale (mean \pm standard deviation) was $1.00 \pm 0.707$ in IJV group and $0.63 \pm 1.061$ in $\mathrm{AxV} / \mathrm{SCV}$ group in $\mathrm{D} 7$ after CVC puncture. The required sample size was calculated using an estimation formula based on the different means between two groups $(20,21)$. Setting the two-sided significance level $(\alpha)$ at 0.05 and statistical power $(1-\beta)$ at 0.8 , a minimum sample size of 90 participants per group (180 participants in total) was estimated to provide sufficient statistical power for detecting the difference between two groups. Considering a $10 \%$ loss to follow-up, we plan to enroll 100 patients in each arm, for a total of 200 patients.

\section{Implantation procedure}

All the procedures will be performed in the out-patient operation room and strictly follow the aseptic principles. Patient's vital signs are monitored continuously using noninvasive blood pressure cuff, electrocardiogram monitor and pulse oximetry. For AxV/SCV group patients, they will be positioned in a neutral supine position on the operation bed while for IJV group patients, they will be positioned in a neutral supine position with the head turned 90 degrees to the left. Two percent lidocaine will be used for local anesthesia. No sedatives will be used for patients during implantable venous access port (IVAP) implantation. Designated brand of infusion ports, BARD (BD, BARD 

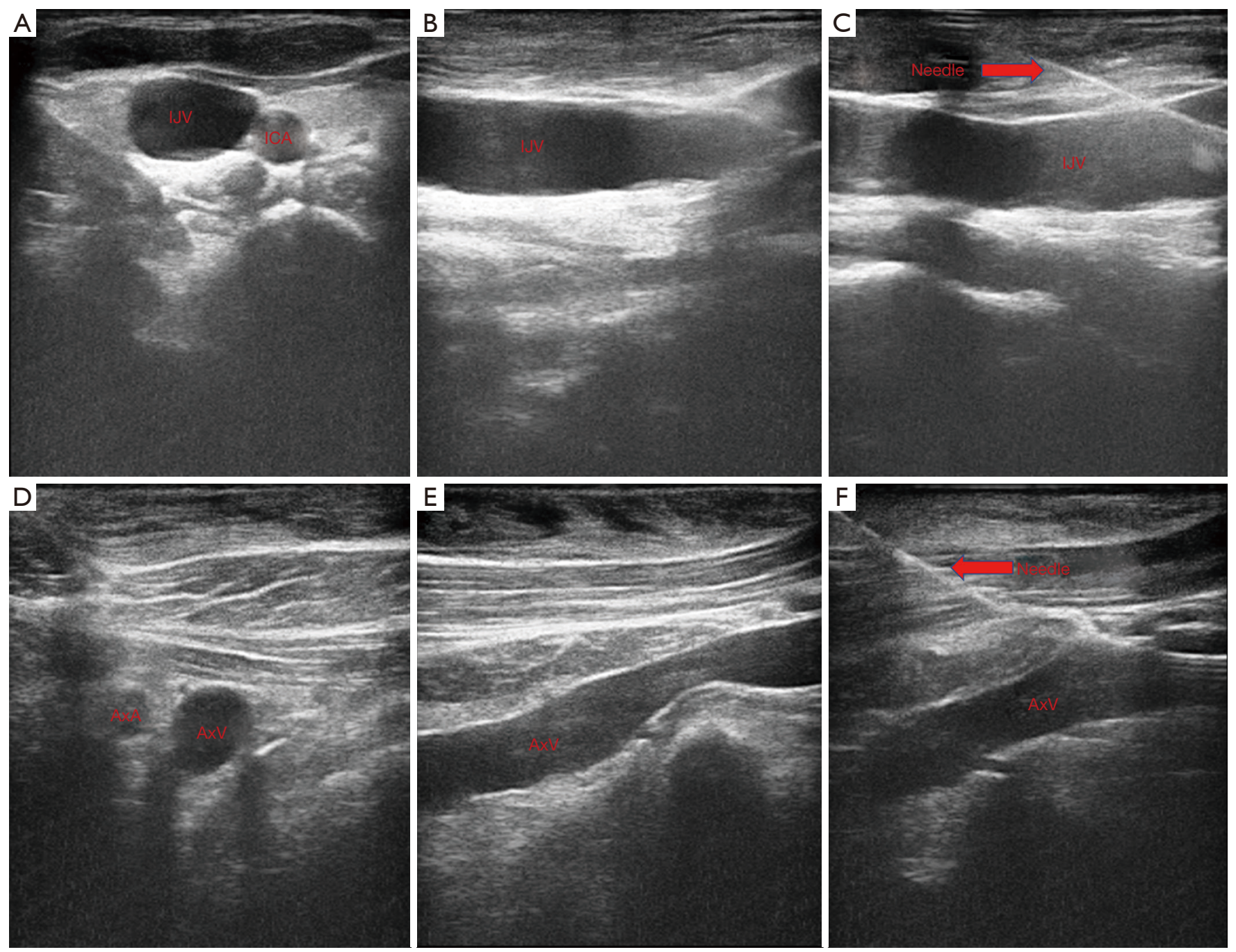

Figure 2 Center venous catheter puncture with ultrasound guidance. (A-C) Center venous catheter puncture through IJV with ultrasound guidance; (D-F) center venous catheter puncture through AxV/SCV with ultrasound guidance. IJV, internal jugular vein; AxV, axillary vein; SCV, subclavian vein; ICA, internal carotid artery; AxA, axillary artery.

Medical Equipment Co., USA) will be used for all patients. Performers will insert the guide wire and catheter with the guidance of ultrasound (MYLAB 30CV, Esaote Medical Technology Co., Ltd., China) for all patients (Figure 2). For IJV group patients, we will insert the guide wire and then replace with catheter through right IJV. For $\mathrm{AxV} /$ SCV group patients, we will insert the guide wire and then replace with catheter through right $\mathrm{AxV}$ or right $\mathrm{SCV}$. All patients are required to perform a chest $\mathrm{X}$-ray right after the catheter insertion (Figure 3) and we will check the incidence of early complications after that.

\section{Outcome measures}

The primary outcome measure of this study is patient comfort at three point-in-time: day 1, just after CVC puncture surgery; day 2, 1 day after CVC puncture and day 7, 1 week after CVC puncture. The interview of patient comfort assessment will be conducted by the researchers face by face on day 1 and conducted with telephones on days 2 and 7. All patients will receive assessment of comfort with a comfort scale table (Table 1) for three times. The comfort scale in our study is a visual analogic scale which 

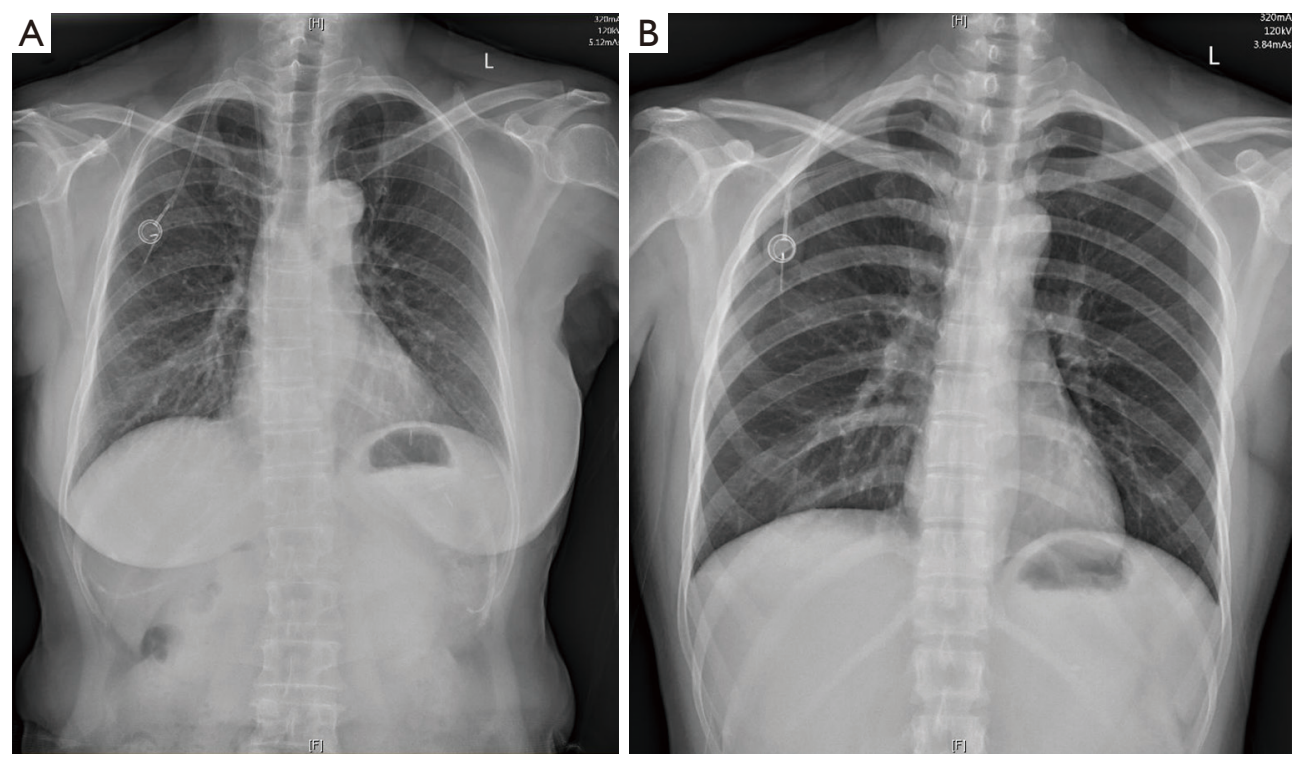

Figure 3 Chest $\mathrm{X}$-rays of patients right after infusion ports implantation. (A) Chest X-ray of patient with center venous catheter puncture through IJV; (B) chest X-ray of patient with center venous catheter puncture through AxV/SCV. IJV, internal jugular vein; AxV, axillary vein; SCV, subclavian vein.

Table 1 Patient comfort scale table

\begin{tabular}{ll}
\hline Grade & \multicolumn{1}{c}{ Discomfort scale } \\
\hline Grade 0 & Without any discomfort \\
Grade 1 & Extremely mild discomfort \\
Grade 2 & A little discomfort \\
Grade 3 & Some discomfort \\
Grade 4 & Rather uncomfortable \\
Grade 5 & Extreme discomfort \\
\hline
\end{tabular}

is divided into six grades of comfort (grade 0: without any discomfort; grade 1: extremely mild discomfort; grade 2: a little discomfort; grade 3: some discomfort; grade 4: rather discomfort; grade 5: extremely discomfort), which has been applied in many medical researches and has been proved to be a simple and effective method to assess patients' comfort or satisfaction $(22,23)$.

Secondary outcome measures of our study are as follows: (I) duration of procedure (minutes), defined as the time between the initial cutaneous sterilization and dressing placement; (II) early complications rate and (III) late complications rate. Early complication is defined as the period of time between intraoperative implantation and the first use of catheter, while late complication is defined as the subsequent period until the infusion port is removed. Early complications include wire advancement difficulties, inadvertent artery puncture, catheter misplacement, pneumothorax or subcutaneous hematoma. Late complications include port related infection, CVC related DVT, and catheter complications such as tip dislocation, catheter rupture or loss of patency. All these complications are defined as following:

(I) Wire advancement difficulties: failure puncture or wire advancement for three times or more;

(II) Inadvertent artery puncture: pulsatile blood reflux through the needle observed during the procedure;

(III) Catheter misplacement: catheter tip identified at any place other than the superior vena cava on the chest X-ray;

(IV) Pneumothorax: characterized by abnormal presence of air in the pleural cavity resulting in the collapse of the lung on the chest X-ray;

(V) Subcutaneous hematoma: characterized by a localized collection of blood, usually clotted, in space near the point of puncture;

(VI) Port related infection: isolation of the same organism from a blood culture and from a semiquantitative or quantitative culture of a catheter segment, accompanied by clinical symptoms of bloodstream infection without any 
other apparent source of infection;

(VII) CVC related DVT: vein thrombosis is defined as thrombosis on the tube wall established by color doppler ultrasound examination;

(VIII) Tip dislocation, catheter rupture: tip dislocation or catheter rupture on the chest $\mathrm{X}$-ray;

(IX) Loss of patency: the infusion port or tube is obstructed and drug cannot pass through the catheter.

\section{Statistical analysis}

All the statistical analysis will be performed with SPSS (IBM SPSS Statistics Version 20.0) and GraphPad Prism5 (GraphPad Software Inc., San Diego, CA, USA). Summary statistics for normally distributed quantitative variables will be expressed as means and standard deviations. For non-normally distributed variables or ordered categorical variable, we use median and interquartile range (IQR). Unordered categorical variables are summarized by ratios and percentages. Differences in means for continuous variables are compared using Student's $t$-test (two groups) and discontinuous variables are compared using Wilcoxon rank test, while differences in proportions are tested by ChiSquare test. $\mathrm{P}$ value below 0.05 is considered statistically significant.

\section{Etbics and informed consents}

This study has been approved by the medical ethics committee of Sun Yat-sen Memorial Hospital, Sun Yatsen University (No. 2020-KY-053). At the same time this study has also been registered on Chinese Clinical Trial Registry (ChiCTR, www.chictr.org.cn) and Chinese Ethics Committee of Registering Clinical Trials (No. ChiCTR2000034986). All patients will sign written informed consents for enrollment and anonymous data publication before randomization.

\section{Discussion}

Before ultrasound guidance was widely used in CVC puncture, SCV and $\mathrm{AxV}$ were less commonly used than IJV because of higher risk of complications such as pneumothorax. After the first attempt of use of ultrasound-guided CVC placement, it has been proved that complications of CVC puncture decrease sharply with the guidance of ultrasound $(7,13)$. As a result, SCV and $\mathrm{AxV}$ routes of access were more and more applied in clinical work in the past years. A retrospective study of 2,586 sequential patients indicated that the $\mathrm{AxV}$ route of access appeared to be a safe and effective alternative to the IJV with the help of ultrasound (10). In our previous clinical work, we found that complication rate patients developed was rather low in both IJV group and AxV/SCV group with ultrasound guidance. Consequently, we strongly recommend the use of ultrasound guidance in CVC placement and all patients enrolled in this study will be performed with ultrasound guidance.

As previously described, almost all the researches comparing IJV and AxV/SCV have focused on the safety and complications of these two insertion approaches while patients' comfort and satisfaction was only involved in few studies $(23,24)$. On the other hand, most studies available are retrospective. In our randomized prospective controlled study, patients' comfort of the infusion ports with long-term CVC is the primary outcome measure while the complications and operation duration are the second outcome measures, which is the first study to put patient's comfort of port in such a high priority. In our preliminary study with a small amount of cases we found that that patients' comfort scale in AxV/SCV group was obviously better than those in IJV group. Most patients in IJV group complained that they felt like there had something against their necks and they were afraid to turn their heads in the several days after the insertion of the CVC. Consequently, we designed this randomized controlled trial to further explore the advantages of IJV group and AxV/SCV group.

There are many factors that may affect patient comfort. To avoid these confounding factors, patients with a history of anxiety or depression will be excluded in our study and all the puncture procedures will be performed by two appointed doctors. Patient's baseline as well as demographic data such age, BMI, chemotherapy types and courses, brands of infusion ports, TNM stage or history of diabetes will all be recorded and reported. At the same time, we will record whether patients take non-steroidal anti-inflammatory drugs (NSAIDs) after CVC puncture to relieve pain, which may also influence patient comfort.

In our protocol, we used a visual analogic scale to access patient comfort. This scale is modified from other papers which were validated $(22,23,25)$. In these previous studies, researchers used a visual analogic scale ranging between 1 (extreme discomfort) and 5 (very comfortable) or an unmarked $10 \mathrm{~cm}$ visual analogic scale from "no discomfort" at $0 \mathrm{~cm}$ to "maximal imaginable discomfort" at 
$10 \mathrm{~cm}$. Although this visual analogic scale is rather simple and reductive, we found it was convenient and easily to be understand by patients. Another study reported an effective assessment tool to access the quality of life with good reliability and validity in BC patients with different implantation sites for totally implanted venous access devices in northern China (26). This scale can effectively access quality of life including aesthetics and privacy, impact on professional activities, social, sports, daily activities and pain, comfort of the treatment, local discomfort, and overall satisfaction, which may be useful for us to take overall assessment of quality of life for patients in future research.

Procedure time of implantation is also an outcome measure in this study. Previous study has demonstrated procedure duration of CVC puncture can be reduced with ultrasound guidance (27). Previous study showed that the access time for the first attempt was significantly shorter in IJV group than in AxV/SCV group (28). However, in our preliminary trial we found that whole procedure duration of AxV/SCV group was shorter than that of IJV group. We think there are several reasons for this difference. Firstly, there is only one incision in AxV/SCV group while there are two incisions in IJV group. Secondly, subcutaneous tunneling is not required in AxV/SCV group during port implantation. Lastly, patient position during operation is much simpler in AxV/SCV group than IJV group. Consequently, the whole procedure time may be longer in IJV group than AxV/SCV group.

Whether the incidence of complications is similar between IJV patients and $\mathrm{AxV}$ or SCV patients still remains controversial. Some studies indicate that the risk of complications such as pneumothorax is higher with SCV approach than IJV approach $(29,30)$. However, other studies demonstrate there is no difference of risk of complications between these different insertion approaches $(10,15)$. A multicenter randomize trial with 3,471 catheters in 3,027 patients from the NEJM demonstrated that SCV catheterization was associated with a lower risk of bloodstream infection and symptomatic thrombosis but a higher risk of pneumothorax than IJV or femoral vein catheterization (31). However, there were no cancer patients or implanted ports in this trial, which was different from our study. Another multicenter, randomized study of 1,484 patients indicated the total complication rate was not significant different between IJV and SCV although more catheter misplacements were observed in SCV group (28). In this study, we will also compare the incidence of complications between these two groups as secondary outcome measures to further evaluate the safety of these different puncture approaches.

In addition to postoperative comfort and complications, postoperative aesthetics is also an important issue. Surgical incision of IJV group patients is visible on the neck obviously, while incision of AxV/SCV group patients is usually sheltered by clothes and will not be observed by others. Although we will not evaluate patients' satisfaction of their surgical incision with a certain scale, we found that many patients especially those young patients in the IJV group complained that the incision was unacceptable with poor cosmetic results. At the same time, we found that patients of $\mathrm{AxV} / \mathrm{SCV}$ group could better cooperate with the doctor's operation and had a more acceptable position during the process, although this is not analyzed as an outcome measure in our study, and this may improve patient comfort and satisfaction during operation.

To the best of our knowledge, this is the first study to compare patient's comfort as primary outcome measure between IJV and AxV/SCV puncture. Also, all included cases are BC patients, which can eliminate bias from different diseases. Last but not least, this is a randomized prospective controlled study and patients' data can be collected completely to analyze. However, there are also some limitations in our study. Firstly, this is not a doubleblind study and may affect patients' subjective feelings. Secondly, we do not evaluate patients' satisfaction to their postoperative aesthetics in this study. Lastly, this is a singlecenter study and the sample size is not large enough; hence, a prospective multicenter survey with larger sample size to compare comfort between IJV puncture and AxV/SCV puncture may be necessary in the future.

\section{Acknowledgments}

The authors wish to thank Dr. Feng-Tao Liu for her guidance on ultrasound scanning in this study and all the patients who are going to participate in this study.

Funding: This study was supported by National Natural Science Foundation of China (81471034) and the Clinical Research Cultivation Project of Sun Yat-sen Memorial Hospital, Sun Yat-sen University.

\section{Footnote}

Reporting Checklist: The authors have completed the SPIRIT reporting checklist. Available at http://dx.doi.org/10.21037/ apm-20-1752 
Peer Review File: Available at http://dx.doi.org/10.21037/ apm-20-1752

Conflicts of Interest: All authors have completed the ICMJE uniform disclosure form (available at http://dx.doi. org/10.21037/apm-20-1752). The authors have no conflicts of interest to declare.

Ethical Statement: The authors are accountable for all aspects of the work in ensuring that questions related to the accuracy or integrity of any part of the work are appropriately investigated and resolved. This study was approved by the Medical Ethics Committee of Sun Yatsen Memorial Hospital, Sun Yat-sen University (No. 2020-KY-053) and was registered at Chinese Clinical Trial Registry (ChiCTR, www.chictr.org.cn) and Chinese Ethics Committee of Registering Clinical Trials (No. ChiCTR2000034986).

Open Access Statement: This is an Open Access article distributed in accordance with the Creative Commons Attribution-NonCommercial-NoDerivs 4.0 International License (CC BY-NC-ND 4.0), which permits the noncommercial replication and distribution of the article with the strict proviso that no changes or edits are made and the original work is properly cited (including links to both the formal publication through the relevant DOI and the license). See: https://creativecommons.org/licenses/by-nc-nd/4.0/.

\section{References}

1. Bray F, Ferlay J, Soerjomataram I, et al. Global cancer statistics 2018: GLOBOCAN estimates of incidence and mortality worldwide for 36 cancers in 185 countries. CA Cancer J Clin 2018;68:394-424.

2. Mincey BA, Palmieri FM, Perez EA. Adjuvant therapy for breast cancer: recommendations for management based on consensus review and recent clinical trials. Oncologist 2002;7:246-50.

3. Sparano JA, Gray RJ, Makower DF, et al. Adjuvant chemotherapy guided by a 21 -gene expression assay in breast cancer. N Engl J Med 2018;379:111-21.

4. Wiegering V, Schmid S, Andres O, et al. Thrombosis as a complication of central venous access in pediatric patients with malignancies: a 5-year single-center experience. BMC Hematol 2014;14:18.

5. Tewari M, Shukla HS. Administration of cancer chemotherapeutic drugs through the enhanced peripheral veins by creating a radiocephalic fistula. Am J Surg 2007;194:240-2.

6. Jan HC, Chou SJ, Chen TH, et al. Management and prevention of complications of subcutaneous intravenous infusion port. Surg Oncol 2012;21:7-13.

7. Gallieni M, Pittiruti M, Biffi R. Vascular access in oncology patients. CA Cancer J Clin 2008;58:323-46.

8. Galloway S, Bodenham A. Ultrasound imaging of the axillary vein--anatomical basis for central venous access. $\mathrm{Br}$ J Anaesth 2003;90:589-95.

9. Sharma A, Bodenham AR, Mallick A. Ultrasound-guided infraclavicular axillary vein cannulation for central venous access. Br J Anaesth 2004;93:188-92.

10. O'Leary R, Ahmed SM, McLure H, et al. Ultrasoundguided infraclavicular axillary vein cannulation: a useful alternative to the internal jugular vein. $\mathrm{Br} \mathrm{J}$ Anaesth 2012;109:762-8.

11. Troianos CA, Hartman GS, Glas KE, et al. Guidelines for performing ultrasound guided vascular cannulation: recommendations of the American Society of Echocardiography and the Society of Cardiovascular Anesthesiologists. J Am Soc Echocardiogr 2011;24:1291-318.

12. Lamperti M, Bodenham AR, Pittiruti M, et al. International evidence-based recommendations on ultrasound-guided vascular access. Intensive Care Med 2012;38:1105-17.

13. Peris A, Zagli G, Bonizzoli M, et al. Implantation of 3951 long-term central venous catheters: performances, risk analysis, and patient comfort after ultrasound-guidance introduction. Anesth Analg 2010;111:1194-201.

14. Matsushima H, Adachi T, Iwata T, et al. Analysis of the outcomes in central venous access port implantation performed by residents via the internal jugular vein and subclavian vein. J Surg Educ 2017;74:443-9.

15. Nagasawa $Y$, Shimizu T, Sonoda H, et al. A comparison of outcomes and complications of totally implantable access port through the internal jugular vein versus the subclavian vein. Int Surg 2014;99:182-8.

16. Agha RA, Altman DG, Rosin D. The SPIRIT 2013 statement--defining standard protocol items for trials. Int J Surg 2015;13:288-91.

17. Chan AW, Tetzlaff JM, Altman DG, et al. SPIRIT 2013 statement: defining standard protocol items for clinical trials. Ann Intern Med 2013;158:200-7.

18. Lan L, Zeng F, Liu GJ, et al. Acupuncture for functional dyspepsia. Cochrane Database Syst Rev 2014;(10):CD008487.

19. Yin W, Pang L, Cao X, et al. Factors associated with 
depression and anxiety among patients attending community-based methadone maintenance treatment in China. Addiction 2015;110 Suppl 1:51-60.

20. Ru Y, Yan XN, Yang SQ, et al. Oral Taodan granules for mild-to-moderate psoriasis vulgaris: protocol for a randomized, double-blind, multicenter clinical trial. Ann Transl Med 2019;7:488.

21. Moher D, Schulz KF, Altman D, et al. The CONSORT statement: revised recommendations for improving the quality of reports of parallel-group randomized trials. JAMA 2001;285:1987-91.

22. Mauri T, Galazzi A, Binda F, et al. Impact of flow and temperature on patient comfort during respiratory support by high-flow nasal cannula. Crit Care 2018;22:120.

23. Babu KG, Suresh Babu MC, Lokanatha D, et al. Outcomes, cost comparison, and patient satisfaction during long-term central venous access in cancer patients: Experience from a Tertiary Care Cancer Institute in South India. Indian J Med Paediatr Oncol 2016;37:232-8.

24. Shafique MN, Akhtar SH, Mahnoor M, et al. Hemodialysis Internal jugular vein versus Subclavian vein Catheters: Complications, patients' comfort, tolerance and cost-effectiveness. Pak J Med Sci 2019;35:124-8.

25. Frat JP, Thille AW, Mercat A, et al. High-flow oxygen through nasal cannula in acute hypoxemic respiratory

Cite this article as: Chen YB, Bao HS, Deng HR, Hu TT, Wen BL, Yi CY, Chen XW, Yan L, Wu JN. Comparison of comfort and complications in breast cancer patients of implantable venous access port (IVAP) with ultrasound guided internal jugular vein (IJV) and axillary vein/subclavian vein (AxV/SCV) puncture: a randomized controlled study protocol. Ann Palliat Med 2020;9(6):4323-4331. doi: 10.21037/apm-20-1752 failure. N Engl J Med 2015;372:2185-96.

26. Liu Y, Xu L, Jiang M, et al. Chinesization of the quality of life assessment, venous device-port, and its reliability and validity tests for patients with breast cancer. J Vasc Access 2020;21:983-9.

27. Orsi F, Grasso RF, Arnaldi P, et al. Ultrasound guided versus direct vein puncture in central venous port placement. J Vasc Access 2000;1:73-7.

28. Shin HJ, Na HS, Koh WU, et al. Complications in internal jugular vs subclavian ultrasound-guided central venous catheterization: a comparative randomized trial. Intensive Care Med 2019;45:968-76.

29. Vinson DR, Ballard DW, Hance LG, et al. Pneumothorax is a rare complication of thoracic central venous catheterization in community EDs. Am J Emerg Med 2015;33:60-6.

30. Björkander M, Bentzer P, Schött U, et al. Mechanical complications of central venous catheter insertions: a retrospective multicenter study of incidence and risks. Acta Anaesthesiol Scand 2019;63:61-8.

31. Parienti JJ, Mongardon N, Mégarbane B, et al. Intravascular complications of central venous catheterization by insertion site. N Engl J Med 2015;373:1220-9. 